\title{
PREFERÊNCIAS ALIMENTARES E DOMICILIAÇÃO DE MOSQUITOS CULICIDAE NO VALE DO RIBEIRA, SÃO PAULO, BRASIL, COM ESPECIAL REFERENCIA A AEDES SCAPULARIS E A CULEX (MELANOCONION)*
}

\author{
Oswaldo Paulo Forattini** \\ Almério de Castro Gomes** \\ Délsio Natal** \\ Iná Kakitani** \\ Daniel Marucci**
}

\begin{abstract}
FORATTINI, O.P. et al. Preferências alimentares e domiciliaçăo de mosquitos Culicidae no Vale do Ribeira, São Paulo, Brasil, com especial referência a Aedes scapularis e a Culex (Melanoconion). Rev. Saúde públ., S. Paulo, 23:9-19, 1989.
\end{abstract}

RESUMO: Apresentam-se novos dados sobre a identificação do sangue ingerido por culicídeos ingurgitados e coletados em quatro localidades do Vale do Ribeira, Estado de São Paulo (Brasil), no período de fevereiro a novembro de 1986 , e que já tinham sido sede de observaçőes anteriores. São fornecidos dados sobre a distribuição de algumas espécies relacionadas ao tipo de ambiente. Focalizam-se $A e$. scapularis e representantes de Culex (Melanoconion), principalmente $C x$. ribeirensis e $C x$. sacchettae. Foi possivel a identificação de 651 repastos sangüineos. Confirmou-se a preferência de Ae. scapularis por mamíferos de grande porte representados por bovinos, equinos e o próprio homem, tendo reagido a todos os anti-soros testados, com exceçăo do correspondente a animais de sangue frio representados por anfíbio. $C x$. ribeirensis revelou resultados que sugerem possível preferência por mamiferos. As duas espécies supracitadas mostram tendência nítida para adaptação ao ambiente modificado pelo homem e capacidade de evolução de seus hábitos de possível domiciliação. Quanto aos outros culicídeos, as coletas de $A n$. bellator, $A$ n. cruzii e $C q$. chrysonotum limitaram-se à isca humana que a segunda dessas espécies rendeu $31,6 \%$ do total de fềmeas capturadas.

DESCRITORES: Mosquitos. Preferências alimentares. Aedes scapularis. Culex. Antropofilia. Domiciliação. Insetos vetores.

\section{INTRODUÇÃO}

Em publicações anteriores foram divulgados os primeiros resultados obtidos na identificação de sangue ingerido e na observaçâo da freqüência domiciliar e endofilia, por parte de mosquitos Culicidae no Vale do Ribeira, Estado de São Paulo, Brasil (Forattini e col.4,5, 1987). Em tais oportunidades, que corresponderam à parte do projeto levada a efeito nos anos de 1984 a 1986 , pôde-se adquirir dados que possibilitaram conhecimento geral sobre hábitos alimentares sangüíneos e de domiciliaridade de mosquitos naquela região. A seqüência das observações implicou a focalização das preferências hematófagas das principais espécies, encontradas em vários ambientes, bem como sua freqüência e distribuição. O relato e a discussão desses novos dados, obtidos no decurso do ano de 1986 , constitui o objetivo do presente trabalho.

\section{MATERIAL E METODO}

As áreas estudadas, juntamente com as técnicas de coleta, de manipulação dos espécimens e de identificação do sangue ingerido já foram, respectivamente, caracterizadas e descritas em trabalhos anteriores ${ }^{4,5}$. No presente trabalho serão mencionadas as áreas onde foram levadas a efeito as atividades, e descritos o ritmo e as modificações introduzidas nos métodos de coleta e de identificação do sangue ingerido.

A presente investigação foi desenvolvida nas áreas e respectivas estações de coleta, relacionadas como segue:

\begin{tabular}{cl} 
Área & \multicolumn{1}{c}{ Estação de coleta } \\
A & Fazenda Experimental \\
B & Bairro de Pariquera-Mirim \\
C & Fazenda Folha Larga \\
D & Área da Fonte (Itapuã)
\end{tabular}
715 - 01255 - Såo Paulo, SP - Brasil. 
Métodos e ritmo das coletas - A experiência anteriormente adquirida permitiu o selecionamento do processo de aspiração e daquele do uso de pequenas redes manuais, como os métodos fundamentados no princípio da busca ativa em possíveis abrigos. Como procedimento baseado na atração passiva, empregou-se a isca humana, utilizada tanto no solo como na copa arbórea das matas, em ambos os casos com tempo de duração operacional correspondente a uma hora e trinta minutos. No primeiro, as coletas eram efetuadas ao longo de trilhas previamente determinadas, como já se descreveu anteriormente ${ }^{4,5}$. Esses vários caminhos corresponderam, fundamentalmente, a três situações, ou seja, interior, margem da mata e terreno aberto.

No que concerne à copa arbórea, as coletas foram efetuadas na plataforma da estação $C$, obviamente de maneira estática e continuadamente, durante o mesmo espaço de tempo já referido.
Em cada área, as capturas obedeceram a ritmo mensal. A coleta ativa foi realizada nos ambientes peri e extradomiciliares. Nestes últimos, foram diferenciadas as áreas de terreno aberto $e$ as de matas onde se discriminou o solo e a margem. No que concerne à isca humana, procedeu-se à sua realização nas mesmas situações, incluindo-se porém a copa arbórea no ambiente florestal.

Essa rotina foi seguida para todas as estaçōes de coleta, exceção feita para o emprego da isca humana na estação $C$, levado a efeito com ritmo bimensal, tanto na situação do terreno aberto como do solo da mata. No que tange à estação $D$, somente foram realizadas capturas no interior da mata, no solo, e por meio de aspiraçào. A Tabela 1 resume o que foi exposto, apresentando os horários, os métodos, os ambientes e as estações onde os trabalhos de coleta foram executados.

TABELA 1

Ritmo mensal de coleta de mosquitos.

\begin{tabular}{|c|c|c|c|}
\hline $\begin{array}{l}\text { Horário } \\
\text { (h) }\end{array}$ & Método & Ambiente & Estação \\
\hline $7: 00-8: 00$ & Aspiração & Peridomicílio & $\mathrm{A}, \mathrm{B}, \mathrm{C}$ \\
\hline $8: 00-12: 00$ & $\begin{array}{l}\text { Aspiração } \\
\text { Rede manual } \\
\text { Isca humana }\end{array}$ & $\begin{array}{l}\text { Aberto } \\
\text { Mata-margem } \\
\text { Mata-solo } \\
\text { Mata-solo } \\
\text { Mata-copa }\end{array}$ & $\begin{array}{l}\mathrm{A}, \mathrm{B}, \mathrm{C}, \mathrm{D}^{*} \\
\mathrm{~A}, \mathrm{~B}, \mathrm{C} \\
\mathrm{C}\end{array}$ \\
\hline $17: 00-21: 00$ & Isca humana & $\begin{array}{l}\text { Mata-copa } \\
\text { Aberto } \\
\text { Mata-margem } \\
\text { Mata-solo }\end{array}$ & $\stackrel{\mathrm{C}}{\mathrm{A}, \mathrm{B}, \mathrm{C}}$ \\
\hline
\end{tabular}

Identificação do sangue ingerido - Nesta etapa, optou-se pela adoção do método capilar (Tempelis e Lofy ${ }^{13}, 1963$ ), para todos os espécimens submetidos a exame. $E$ isso em face de maior economia de tempo e de reagentes que essa técnica propicia. Foram utilizados anti-soros para os mesmos grupos de fontes anteriormente focalizados. Houve redução do número daqueles referentes a mamiferos, que foram limitados a bovinos (Bos), eqüino (Equus), homem/primata (Homo), marsupial (Didelphis) e roedor (Rattus).

Os resultados conseguidos nas observaçð̃es anteriores permitiram o selecionamento inicial de alguns culicideos que, do ponto de vista epidemiológico, revelaram maior significância, se- ja atual ou potencial. Dessa maneira, foram consideradas 10 espécies ou grupos. Estes, correspondendo a duas ou mais categorias específicas cujas fêmeas dificilmente se distinguem pelos seus caracteres morfológicos. Assim pois, foram selecionados, Aedes scapularis, grupo serratus (compreendendo Ae. hastatus, Ae. nubilus, Ae. oligopistus e Ae. serratus), Anopheles cruzii, Coquillettidia chrysonotum, Cq. venezuelensis, grupo coronator (compreendendo Culex coronator e CX. usquatus), Culex ribeirensis, Cx. sacchettae, Psorophora albigenu e $P s$. ferox. Contudo, em face do predomínio de Ae. scapularis nos resultados obtidos, as atenções concentraram-se principalmente nos dados referentes a esse mosquito. 


\section{RESULTADOS}

No período compreendido de fevereiro a novembro de 1986, foram coletados 70.602 mosquitos, dos quais 61.390 fêmeas e $9.212 \mathrm{ma}$ chos. Do total de espécimens femininos, 59.849 $(97,5 \%)$ foram representantes de espécies ou grupos mais freqüentes, entendendo-se como tal todo aquele que tivesse comparecido nas coletas com, pelo menos, 100 exemplares, ao longo do mencionado espaço de tempo. Em relação a estes mosquitos, o rendimento dos métodos de captura foram de $70,7 \%$ para a isca hu- mana, $19,1 \%$ para a aspiração e de $10,2 \%$ para a rede manual. A Tabela 2 apresenta, em números totais, a relação dos resultados dessas coletas. Pode-se verificar que o emprego de procedimentos que se basearam no princípio da busca ativa (aspiração e rede manual) forneceram, em seu conjunto, $29,3 \%$ das fêmeas, enquanto o restante dos espécimens desse sexo foi conseguido mediante a utilização da isca humana.

No que concerne aos mosquitos selecionados para o estudo de possíveis preferências alimentares mediante a identificação do sangue ingerido, o conjunto do material coletado foi de

\section{TABELA 2}

Distribuição numérica das fêmeas de Culicídeos, obtidas com maior frequiència nos vários métodos de coleta, e apresentadas em ordem decrescente (período de fevereiro a novembro de 1986).

\begin{tabular}{|c|c|c|c|c|}
\hline $\begin{array}{l}\text { Métodos de coleta } \\
\text { Espécies * }\end{array}$ & Aspiração & $\begin{array}{l}\text { Rede } \\
\text { manual }\end{array}$ & $\begin{array}{c}\text { Isca } \\
\text { humana }\end{array}$ & Total \\
\hline $\begin{array}{l}\text { Anopheles cruzii } \\
\text { Grupo serratus(a) } \\
\text { Aedes scapularis } \\
\text { Culex nigripalpus } \\
\text { Anopheles bellator } \\
\text { Coquillettidia chrysonotum } \\
\text { Psorophora ferox } \\
\text { Phoniomyia sp. } \\
\text { Culex (Culex) sp. } \\
\text { Uranotaenia lowii } \\
\text { Cx. ribeirensis } \\
\text { Cq. venezuelensis } \\
\text { Cx. sacchettae } \\
\text { Cx. mollis } \\
\text { Mansonia (Mansonia) sp. } \\
\text { Psorophora albigenu } \\
\text { Ae. fulvus } \\
\text { Grupo coronator(b) } \\
\text { Grupo flavisetosus(c) } \\
\text { Limatus durhami } \\
\text { Cx. declarator } \\
\text { Wyeomyia sp. } \\
\text { Wyeomyia confusa } \\
\text { Ps. lutzii } \\
\text { Culex (Melanoconion) sp. } \\
\text { Runchomyia reversa } \\
\text { Culex (Microculex) sp. } \\
\text { Cx. pedroi } \\
\text { An. albitarsis } \\
\text { Ur. geometrica } \\
\text { Cx. oedipus } \\
\text { Outras espécies(d) }\end{array}$ & $\begin{array}{r}36 \\
1.729 \\
1.712 \\
1.657 \\
3 \\
60 \\
216 \\
109 \\
1.181 \\
1.696 \\
106 \\
89 \\
69 \\
694 \\
21 \\
153 \\
17 \\
371 \\
29 \\
48 \\
246 \\
37 \\
42 \\
118 \\
215 \\
7 \\
154 \\
111 \\
5 \\
119 \\
119 \\
271\end{array}$ & $\begin{array}{r}45 \\
1.496 \\
1.555 \\
455 \\
3 \\
74 \\
506 \\
357 \\
493 \\
- \\
13 \\
7 \\
15 \\
233 \\
10 \\
80 \\
18 \\
43 \\
96 \\
102 \\
57 \\
71 \\
69 \\
109 \\
17 \\
14 \\
19 \\
25 \\
- \\
1 \\
- \\
142\end{array}$ & $\begin{array}{r}19.388 \\
4.105 \\
3.019 \\
1.319 \\
2.664 \\
2.326 \\
1.188 \\
1.294 \\
35 \\
5 \\
1.153 \\
1.154 \\
963 \\
38 \\
840 \\
494 \\
419 \\
22 \\
310 \\
218 \\
1 \\
151 \\
143 \\
25 \\
16 \\
159 \\
- \\
36 \\
139 \\
1 \\
1 \\
658\end{array}$ & $\begin{array}{r}19.469 \\
7.330 \\
6.286 \\
3.431 \\
2.670 \\
2.460 \\
1.910 \\
1.760 \\
1.709 \\
1.701 \\
1.272 \\
1.250 \\
1.047 \\
965 \\
871 \\
727 \\
454 \\
436 \\
435 \\
368 \\
304 \\
259 \\
254 \\
252 \\
248 \\
180 \\
173 \\
172 \\
144 \\
121 \\
120 \\
1.071\end{array}$ \\
\hline Total & 11.440 & 6.125 & 42.284 & 59.849 \\
\hline
\end{tabular}

a - Ae. hastatus, Ae. nubilus, $A$. oligopistus e Ae. serratus.

$\mathrm{b}-\mathrm{Cx}$. coronator e $C x$. usquatus

c - Li. asuleptus e Li. flavisetosus

d - com os números totais entre parênteses: Ae, perventor e Ae. hortator (149);An. mediopunctatus (103); Anopheles sp. (152); Cq. hermanoi (117); Cx. (Mel.) grupo pilosus (148); Ph. davisi (138); Trichoprosopon sp. (140); Wy. aporonoma (124).

* Até o mínimo total de 100 exemplares no período. 
42.187 exemplares. Isso representou $68,7 \%$ do global de fêmeas capturadas, e $70,5 \%$ dos culicídeos considerados como mais freqüentes, de acordo com o critério já exposto (Tabela 2). Dentro dessas espécies foram obtidas 1.270 $(2,1 \%)$ fêmeas ingurgitadas, $780(1,3 \%)$ das quais se encontravam em condições satisfatórias para serem submetidas aos testes de precipitinas. Destas, $696(1,2 \%)$ constituíram os representantes dos mosquitos já mencionados e $84(0,1 \%)$ os de outras espécies.

Os resultados globais de identificação das fontes sangüíneas encontram-se na Tabela 3 , onde se pode verificar que $85,5 \%$ dos mosquitos ingurgitados e testados revelaram-se reagentes. O maior número deles foi de representantes de Ae. scapularis, responsável por 338 resultados positivos dentre o total de 595 conseguidos, ou seja, $56,8 \%$ dos espécimens reagentes. Quanto aos restantes 84 exemplares testados, obtiveram-se 49 que mostraram positividade. Os resultados observados foram os seguintes:

\section{Espécie(s)}

\section{Aedes fulvus}

Anopheles bellator

grupo intermedius (An. api-

cimacula e An. intermedius)

Culex bastagarius

Cx. nigripalpus

$C x$. oedipus

Mansonia (Mansonia) sp.

Sorophora cingulato

\section{Ps. discrucians \\ Ps. lutzii \\ Uranotaenia lowii}

Total

\begin{tabular}{cl} 
N. & \multicolumn{1}{c}{ Fonte (s) } \\
1 & homem \\
1 & homem \\
3 & bovino (2); \\
& eqüino \\
1 & equiino \\
1 & bovino \\
1 & anfíbio \\
1 & homem \\
34 & bovino (22); \\
& equiino (11); \\
1 & misto \\
3 & homem \\
& home $(2) ;$ \\
2 & homem bovino
\end{tabular}

49
Todos os demais mosquitos examinados resultaram negativos para esse hospedeiro. Assim sendo, considerando os seis testes restantes, a positividade, por número de fontes, entre as espécies principais, distribuiu-se da maneira seguinte:
N. de fontes

6

4

3
2
1

\section{Espécie(s)}

Aedes scapularis; grupo serratus
Coquillettidia chrysonotum; Cq.
venezuelensis; Culex ribeirensis;
Psorophore albigenu
Cx. sacchettae
grupo coronator
Anopheles cruzii; Psorophora
ferox

Dos mosquitos coletados com isca humana, apenas 153 espécimens puderam ser submetidos a exame, 143 dos quais mostraram-se reagentes. Como era de se esperar, a maioria, representada por 121 exemplares, forneceu reação simples para sangue humano, e $22(15,4 \%)$ mostraram outras fontes, incluido um espécimen com reação mista, positiva também para homem. Isso significa que esses mosquitos tiveram oportunidade de sugar outros hospedeiros antes de se aproximarem da isca humana onde, presumivelmente, iriam completar o seu repasto. Tal positividade distribuiu-se da seguinte maneira:

$\begin{array}{lcccc}\quad \text { Espécie(s) } & \text { ave } & \text { bovino } & \text { equiino } & \text { mista } \\ \text { Aedes scapularis } & - & 9 & 2 & - \\ \text { grupo serratus } & 6 & - & - & 1^{*} \\ \text { Culex nigripalpus } & - & 1 & - & - \\ \text { Psorophora albigenu } & - & 1 & - & - \\ \text { Ps. lutzii } & - & 2 & - & - \\ \quad \text { Total } & 6 & 13 & 2 & 1 \\ \text { * ave + bovino + homem } & & \end{array}$

Nas coletas levadas a efeito na copa arbórea florestal obtiveram-se 8.846 espécimens femininos das espécies mais freqüentes, como segue:

\begin{tabular}{lr}
\multicolumn{1}{c}{ Espécie (s) } & N. \\
Anopheles cruzii & 6.620 \\
An. bellator & 844 \\
Phoniomyia sp. & 634 \\
Culex nigripalpus & 256 \\
Psorophora ferox & 157 \\
Aedes scapularis & 119 \\
Cx. sacchettae & 114 \\
grupo serratus & 102 \\
\multicolumn{1}{c}{ Total } & 8846
\end{tabular}




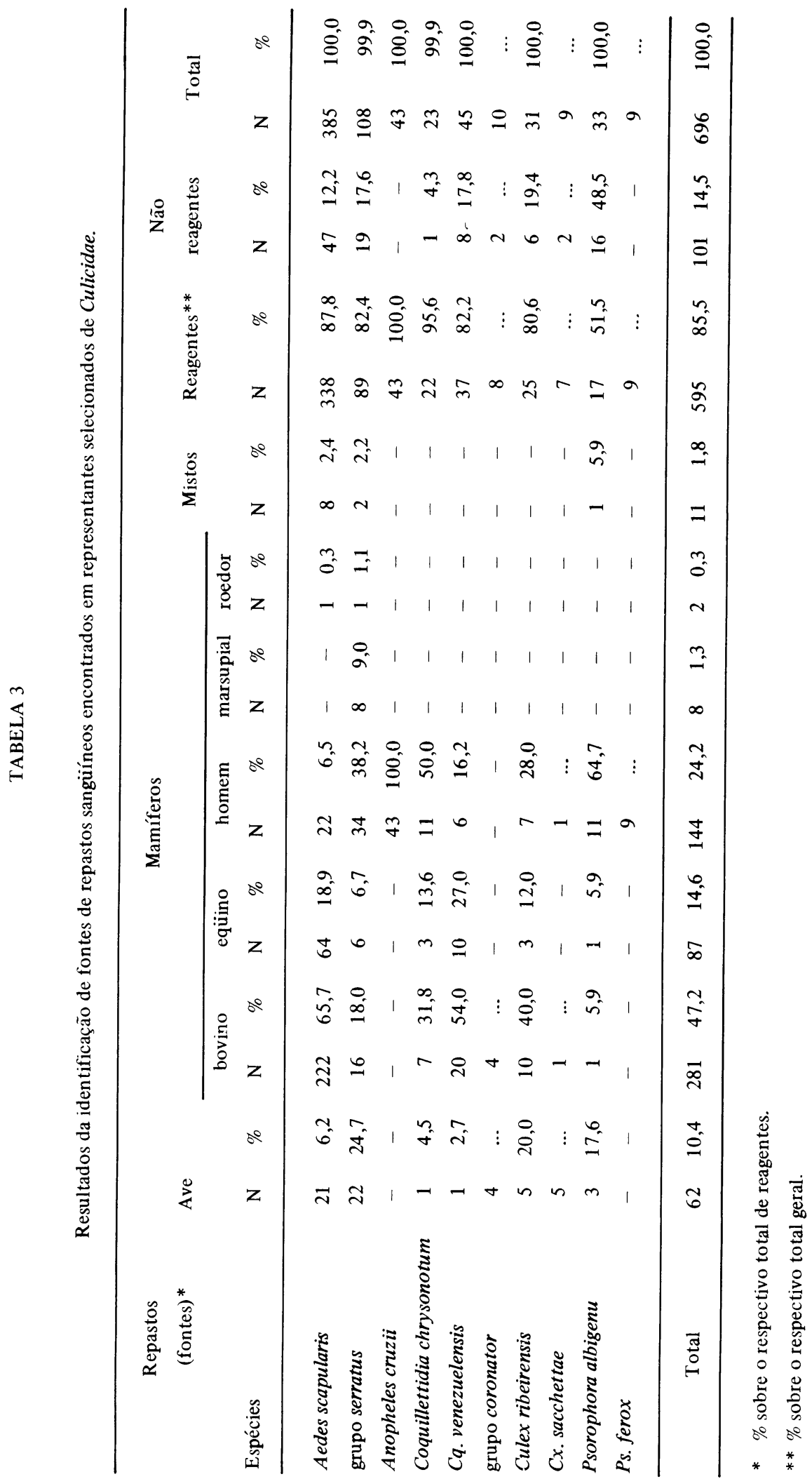


TABELA 4

Resultados da identificação de repastos sangüíneos mistos encontrados em espécies de Culicidae.

\begin{tabular}{|c|c|c|}
\hline Repastos (fontes) & N. & Espécie(s) \\
\hline $\begin{array}{l}\text { Duplas: } \\
\text { ave + bovino } \\
\text { ave + marsupial } \\
\text { bovino + equiino } \\
\text { bovino + homem } \\
\text { eqüino + h omem }\end{array}$ & $\begin{array}{l}3 \\
1 \\
2 \\
3 \\
1\end{array}$ & $\begin{array}{l}\text { Aedes scapularis (2); Psorophora cingulata } \\
\text { Aedes scapularis } \\
\text { Aedes scapularis } \\
\text { Aedes scapularis (2); Grupo serratus } \\
\text { Aedes scapularis }\end{array}$ \\
\hline $\begin{array}{l}\text { Triplas: } \\
\text { ave }+ \text { bovino }+ \text { h omem } \\
\text { ave }+ \text { equino }+ \text { h omem }\end{array}$ & $\begin{array}{l}1 \\
1\end{array}$ & $\begin{array}{l}\text { Grupo serratus } \\
\text { Psorophore albigenu }\end{array}$ \\
\hline Total & 12 & \\
\hline
\end{tabular}

Apenas 18 mosquitos puderam ser submetidos a exame, 17. dos quais com resultados positivos. Destes, 16 An. cruzii reagiram para sangue humano, e dois do grupo serratus reagiram, um para o mesmo hospedeiro e o outro para ave.

A espécie Ae. scapularis propiciou a obtenção de 346 identificações de fontes alimentares. A distribuição dessa positividade, pelos ambientes onde foram efetuadas as coletas, encontra-se na Tabela 5. Ali também se discrimina os vários rendimentos de acordo com o mesmo critério, representados pelos números totais de fêmeas capturadas.

No que concerne a outras espécies, potencialmente importantes sob o ponto de vista epidemiológico, há que se referir os dados obtidos para os representantes de Culex (Melanoconion). $\mathrm{O}$ número de fêmeas desse grupo, que compareceram às capturas com o emprego dos três métodos, foi de 3.556. Isso representou $5,8 \%$ do total obtido e referente a esse sexo. Tais dados acham-se expostos na Tabela 6. Nas coletas com a isca humana destacaram-se $C x$. sacchettae com $92,0 \%, C x$. ribeirensis com $90,6 \%$ e $C x$. taeniopus com $77,3 \%$, percentuais esses referentes ao número total de respectivos representantes coletados. Em seu conjunto, esses três culicídeos constituíram $95,3 \%$ dos exemplares do mesmo subgênero que foram supostamente atraídos pela isca humana.

Quanto ao material conseguido mediante o uso da aspiração e das redes manuais, as mesmas supracitadas espécies perfizeram $17,0 \%$ do total de representantes de Melanoconion capturados. De maneira global, eles foram responsáveis por $67,1 \%$ do total de fêmeas coletadas e desse subgênero.

Ainda em relação aos culicídeos desse subgênero, as coletas feitas no ambiente peridomiciliar forneceram os resultados apresentados na Tabela 7. Ali encontram-se registrados não apenas os resultados numéricos, como também os das reações positivas obtidas na identificação do sangue ingerido, e o rendimento nas três estações de captura. Apesar do pequeno número

TABELA 5

Distribuição espacial das reaçōes positivas para vários hospedeiros observadas em Aedes scapularis.

\begin{tabular}{|c|c|c|c|c|c|c|c|c|c|c|c|c|c|c|c|c|}
\hline $\begin{array}{l}\text { Reaçōes* } \\
\text { Ambiente }\end{array}$ & $\stackrel{A}{A}$ & & $\begin{array}{l}\text { Bov } \\
\text { AR }\end{array}$ & $\begin{array}{c}\text { no } \\
\text { IH }\end{array}$ & $\begin{array}{l}\text { Eqi } \\
\text { AR }\end{array}$ & $\begin{array}{l}\text { ino } \\
\text { IH }\end{array}$ & $\begin{array}{l}\text { Hon } \\
\text { AR }\end{array}$ & IH & $\begin{array}{l}\text { Marst } \\
\text { AR }\end{array}$ & $\begin{array}{c}\text { upial } \\
\text { IH }\end{array}$ & $\begin{array}{l}\text { Roe } \\
\text { AR }\end{array}$ & $\begin{array}{l}\text { dor } \\
\text { IH }\end{array}$ & $\begin{array}{l}\text { Tota } \\
\text { reaç } \\
\text { AR }\end{array}$ & $\begin{array}{l}\text { al de } \\
\text { IH }\end{array}$ & $\mathbf{T}$ & $\begin{array}{l}\text { Total de } \\
\text { espécimens } \\
\text { coletados }\end{array}$ \\
\hline $\begin{array}{l}\text { Mata - solo } \\
\text { Mata - copa } \\
\text { Mata - margem } \\
\text { Aberto } \\
\text { Peridomicílio }\end{array}$ & $\begin{array}{c}15 \\
- \\
- \\
- \\
10\end{array}$ & $\begin{array}{l}- \\
- \\
- \\
-\end{array}$ & $\begin{array}{r}118 \\
- \\
4 \\
36 \\
61\end{array}$ & $\begin{array}{l}9 \\
- \\
- \\
- \\
-\end{array}$ & $\begin{array}{r}42 \\
- \\
1 \\
9 \\
15\end{array}$ & $\begin{array}{l}1 \\
- \\
- \\
-\end{array}$ & $\begin{array}{c}6 \\
-6 \\
- \\
2\end{array}$ & $\begin{array}{l}4 \\
- \\
2 \\
2 \\
-\end{array}$ & $\begin{array}{l}1 \\
- \\
- \\
- \\
-\end{array}$ & $\begin{array}{l}- \\
- \\
- \\
-\end{array}$ & $\begin{array}{l}1 \\
- \\
- \\
- \\
-\end{array}$ & $\begin{array}{l}- \\
- \\
- \\
-\end{array}$ & $\begin{array}{c}183 \\
- \\
11 \\
45 \\
88\end{array}$ & $\begin{array}{c}14 \\
- \\
2 \\
3 \\
-\end{array}$ & $\begin{array}{c}197 \\
- \\
13 \\
48 \\
88\end{array}$ & $\begin{array}{r}2.752 \\
119 \\
1.186 \\
1.120 \\
331\end{array}$ \\
\hline Total & 25 & - & 219 & 9 & 67 & 2 & 14 & 8 & 1 & - & 1 & - & 327 & 19 & 346 & 5.508 \\
\hline
\end{tabular}

* AR - métodos da aspiração e do uso da rede manual IH - isca humana 


\section{TABELA 6}

Distribuição numérica das fêmeas de Culex (Melanoconion), obtidas nos vários métodos de coleta (período de fevereiro a novembro de 1986).

\begin{tabular}{|c|c|c|c|c|}
\hline Métodos de coleta & Aspiraçāo & $\begin{array}{l}\text { Rede } \\
\text { Manual }\end{array}$ & $\begin{array}{l}\text { Isca } \\
\text { Humana }\end{array}$ & Total \\
\hline Cx. aliciae & 8 & - & 2 & 10 \\
\hline Cx. eureonotatus & 12 & - & 2 & 14 \\
\hline Cx. bastagarius & 52 & - & 2 & 54 \\
\hline Cx. delpontei & 17 & - & 1 & 18 \\
\hline Cx. evansae & 33 & - & 3 & 36 \\
\hline Cx. lopesi & 7 & 2 & 1 & 10 \\
\hline Cx. misionensis & 5 & 1 & $i$ & 7 \\
\hline Cx. ocellatus & 2 & 2 & - & 4 \\
\hline Cx. ocossa & 11 & 1 & 1 & 13 \\
\hline Cx. plectoporpe & 99 & - & - & 99 \\
\hline Cx. (Mel.) sp. grupo atratus & 15 & 2 & 5 & 22 \\
\hline$C x$. (Mel.) sp. grupo intrincatus & 54 & 8 & 11 & 73 \\
\hline Cx. (Mel.) sp. grupo pilosus & 123 & 24 & 1 & 148 \\
\hline Cx. spissipes & 41 & 12 & 14 & 67 \\
\hline Cx. taeniopus & 9 & 6 & 51 & 66 \\
\hline Cx. theobaldi & 5 & 1 & 3 & 9 \\
\hline Cx. zeteki & 41 & - & 6 & 47 \\
\hline Outras* & 620 & 70 & 2.169 & 2.859 \\
\hline Total & 1.154 & 129 & 2.273 & 3.556 \\
\hline
\end{tabular}

*Cx. oedipus; $C x$. pedroi; $C x$. ribeirensis; $C x$. sacchettae; $C x$. (Melanoconion) sp.

A té o mínimo total de 100 exemplares no período.

\section{TABELA 7}

Distribuição numérica das fêmeas de Culex (Melanoconion) coletadas no ambiente peridomiciliar e dos resultados obtidos na identificação de fontes para o sangue ingerido.

\begin{tabular}{|c|c|c|c|c|c|c|}
\hline \multirow{2}{*}{ Espécies } & \multirow{2}{*}{$\mathbf{N}$} & \multicolumn{3}{|c|}{ Reaçōes positivas } & \multicolumn{2}{|c|}{ Estaçōes } \\
\hline & & hospedeiro(s) & n. & fonte (s) & A, B & C \\
\hline$C x$. aureonotatus & 10 & - & - & & 10 & - \\
\hline Cx. bastagarius & 48 & - & - & & 48 & - \\
\hline Cx. delpontei & 9 & - & - & & 9 & - \\
\hline Cx. evensae & 25 & - & - & & 25 & - \\
\hline Cx. oedipus & 67 & 1 & 1 & anfíbio & 27 & 40 \\
\hline Cx. pedroi & 20 & - & - & & 20 & - \\
\hline Cx. plectoponpe & 18 & - & - & & 8 & 10 \\
\hline Cx. ribeirensis & 55 & 3 & 12 & ave (2), bovino (9), & 53 & 2 \\
\hline$C x$. sacchettae & 35 & 2 & 4 & ave (3), bovino & 28 & 7 \\
\hline Cx. (Mel.) sp. grupo intrincatus & 11 & - & - & & 9 & 2 \\
\hline Cx. (Mel.) sp. grupo pilosus & 34 & - & - & & 10 & 24 \\
\hline Cx. spissipes & 9 & - & - & & 9 & - \\
\hline Cx. zetekt & 17 & - & - & & 16 & 1 \\
\hline Outras espécies* & 94 & - & - & & 94 & - \\
\hline Total & 452 & 6 & 17 & $\begin{array}{l}\text { anfíbio; ave (5); } \\
\text { bovino }(10) \text {; } \\
\text { equiino }\end{array}$ & 366 & 86 \\
\hline
\end{tabular}

* $C x$. alicibe (5); $C x$. lopesi (2); $C x$. misionensis (3); $C x$. ocossa (5); $C x$. (Mel.) grupo atratus (5); $C x$. theobaldi (4); $C x$. (Melanoconion) sp. (70).

de reações sorológicas, que foi possivel fazer, pode-se observar a utilização de quatro fontes alimentares por parte desses mosquitos, com predominio para bovino e aves, além da participação praticamente restrita a $C x$. ribeirensis e $C x$. sacchettae. No que tange a todos os repre- 
sentantes encontrados nesse ambiente, os dados globais permitiram verificar o predomínio de $C x$. oedipus, $C x$. ribeirensis, $C x$. bastagarius e $C x$. sacchettae, pela ordem e como espécies individualizadas na identificação.

A Tabela 7 mostra também os resultados concernentes ao peridomicilio nas três estações, A, B e C (Tabela 1). Tais dados correspondem aos dois tipos fundamentais de meio ambiente geral, ou seja, alterado e com atividade agropecuária intensa para as A e B, e preservado ou primitivo com reduzida atividade agropecuária para a C. Como se pode verificar, o rendimento foi sensivelmente maior nas duas primeiras, tanto em número de espécies como de exemplares.

\section{DISCUSSÃO}

Em sequiência às discussões anteriores (Forattini e col. $.4,5$ 1987), são discutidos no presente trabalho aspectos que se tenham distinguido dos já observados, bem como possíveis novos dados e confirmações dos registrados naquelas oportunidades.

De início, confirma-se que as técnicas de procura ativa, principalmente a de aspiração, forneceram a maioria do material submetido a exame, ou seja, $87,4 \%$ dos espécimens, contra $19,6 \%$ dos obtidos com o procedimento passivo da coleta com isca humana. Contudo, a justificativa do uso deste residiu no interesse epidemiológico do comportamento de mosquitos que procuram o homem para completar o seu repasto sangüíneo, tendo anteriormente se alimentado em outros hospedeiros. Essa informação permite orientar a observação sobre possível ecletismo alimentar e, assim, a capacidade potencial de veiculação de agentes infecciosos por parte desses culicídeos. Nesse sentido, confirmou-se a possibilidade desse papel para $A e$. scapularis, mostrando reações positivas para todos os anti-soros testados, exceção feita para o de anfíbio, e com expressivo número de reações mistas (Tabelas 3 e 4). De maneira geral, esse mosquito reafirmou sua preferência por sangue de mamíferos, uma vez que das 346 reações positivas, obtidas com espécimens ingurgitados, $87,2 \%$ o foram para esses hospedeiros, sendo de $86,7 \%$ o percentual correspondente aos mamíferos de grande porte representados por bovinos, equuinos e o próprio homem. Acresce considerar que, de maneira geral, essa espécie foi representada pela maioria dos exemplares ingurgitados de mosquitos que foram coletados, submetidos a exame, e com resultados positivos para as técnicas empregadas na identificação do sangue ingerido.
O predomínio de mamíferos de porte, especialmente bovinos, nas fontes sangüíneas de Ae. scapularis, provavelmente possa ter sido decorrência, pelo menos em parte, da abundância desses animais nas áreas onde foram efetuadas as coletas. Tal hipótese encontra apoio nos resultados semelhantes, observados com outros mosquitos, principalmente Coquillettidia chrysonotum, Cq. venezuelensis e Culex ribeirensis. Esse aspecto torna-se particularmente ponderável para as estações A e B, onde a atividade agropecuária é intensa. No entanto, sendo de se esperar tal resultado no material coletado nos ambientes peridomiciliar e aberto, o mesmo não se poderia aventar relativamente ao achado dessa positividade em espécimens coletados no ambiente representado pelo interior das matas (Tabela 5). Aparentemente, é de se levantar a hipótese de fêmeas terem abandonado esse ambiente com a finalidade da busca de fontes para alimentação, ou então, terem-no procurado como local de abrigo para repouso pós-prandial, ou ambos os comportamentos. De qualquer maneira, nos meios representados pela mata-solo e mata-margem, foram obtidas $58,9 \%$ das reações positivas para o conjunto constituído por sangue de bovino e eqüino em $A e$. scapularis. As restantes, ainda para esse mosquito, foram conseguidas no aberto e no peridomicilio. Chama também atenção a reatividade observada para sangue de ave que esse culicídeo apresentou. Houve positividade na mata-solo, e que voltou a ser encontrada em apenas mais um ambiente, que foi o do peridomicílio. Esta última, muito provavelmente, refletiu a utilização de aves domésticas, e aquela, a de aves silvestres. Tais resultados, relativos a esse culicídeo, concordam, de maneira geral, com os observados em outras regiões desta parte meridional da América do Sul (Mitchell e col. ${ }^{7.8 .9}, 1985,1987$; Oliveira e Heyden ${ }^{11}$, 1986; Guimarães e col. ${ }^{6}$, 1987).

No que concerne à possivel domiciliação de Ae. scapularis, os resultados dessas observações, embora restritas ao ambiente peridomiciliar, concordam com os anteriormente relatados para as mesmas localidades (Forattini e col. $\left..^{5}, 1987\right)$. A atração das fontes de alimentação existentes no peridomicilio certamente exercem apreciável influência na aproximação das fêmeas. No entanto, esses espécimens também acusaram a presença de sangue humano, embora em baixa proporção $-2,3 \%-$, se comparada com as das reaçōes registradas para outros hospedeiros (Tabela 5). É lícito pois, mais uma vez, admitir a potencial endofilia desse mosquito, mercê da qual poderá vir a desenvolver há- 
bitos domiciliados, mais acentuados, pelo menos nesta região sul-americana.

Em relação aos representantes de Culex ( $M e$ lanoconion), os resultados obtidos com os três métodos de coleta revelaram também maior rendimento com a isca humana (Tabelas 2 e 6). Todavia, da apreciável variedade de espécies encontradas, a distribuição relativa ao ambiente do peridomicílio mostrou predomínio em áreas sujeitas a mais intensa alteração de origem antrópica, ou seja, as representadas, na presente pesquisa, pelas estações A e B. Nelas, tornou-se evidente a atividade de $C x$. ribeirensis e $C x$. sacchettae, confirmando quadro já anteriormente descrito (Forattini e col. $.^{5}, 1987$ ). Ao que tudo indica, e à semelhança do que ocorre com Ae. scapularis, não se pode descartar a hipótese de aproximação domiciliar por parte desses mosquitos estimulada pela presença de animais domésticos habitantes do peridomicílio (Tabela 7). Em que pese o pequeno número de reações positivas ali conseguidas, nota-se a alimentação nessas fontes, principalmente bovinos. Em relação a $C x$. ribeirensis, tais resultados levam a reforçar a hipótese de sua capacidade de sobrevivência e de desenvolvimento no ambiente modificado pelo homem (Forattini e col. ${ }^{3}, 1986$; Forattini e Gomes ${ }^{2}, 1988$ ).

Ainda no que concerne a representantes de Culex (Melanoconion), as observaçōes levadas a efeito na região setentrional da Argentina têm revelado a possibilidade de $C x$. delpontei ser responsabilizado pela transmissão enzoótica de arbovírus locais, como o da encefalite eqüina venezuelana (Mitchell e col. ${ }^{7,9}, 1985,1987$ ). Na presente investigação, foi assinalada a presença dessa espécie na região. Todavia, seu comparecimento foi discreto, limitado a poucas fêmeas, a maioria coletada por aspiração. Contudo, boa parte dessa pequena amostra foi encontrada no ambiente peridomiciliar, e todos os espécimens foram conseguidos em áreas de ambiente alterado e com atividade agropecuária intensa (Tabelas 6 e 7). Tais fatos ensejam a oportunidade da realização de novas observações, objetivando investigar o potencial de adaptação desse mosquito ao ambiente artificial do homem e sua eventual tendência à domiciliação.

Quanto às outras espécies, embora a propensão de se aproximarem da isca humana tenha sido preponderante para a maioria delas, algumas revelaram reduzida tendência nesse sentido. A Tabela 2 mostra os resultados obtidos com as coletas de Culex declarator, Cx. mollis, Cx. oedipus, Psorophora lutzii, Uranotaenia geometrica e Ur. lowii, com aparente pouca freqüência à essa isca quando comparada com as dos outros métodos de captura empregados.
Aliás, esse aspecto já foi assinalado em outras regioes, para alguns desses mosquitos (Oliveira $^{10}, 1984$; Roberts e col. ${ }^{12}, 1985$; Oliveira e Heyden "1, 1986). Por outro lado, chama a atenção o comportamento de Anopheles bellator, An. cruzii e $C q$. chrysonotum, com rendimento praticamente limitado à isca humana e na elevada cifra de $31,6 \%$, do total de fêmeas coletadas na presente pesquisa, para a segunda dessas espécies. Merece atenção também os dados referentes a $C x$. nigripalpus, revelando comparecimento significante tanto na aspiração como na isca humana. Isso sugere a existência de hábitos ubiqüistas para esse culicídeo, os quais também já foram registrados em outras regiōes (Degallier e col. ${ }^{1}, 1978$; Oliveira ${ }^{10}, 1984$; Guimarães e col..$^{6}$, 1987).

\section{CONCLUSÕES}

Como decorrência dos resultados apresentados e das consideraçðes feitas, conclui-se:

1 - Reforçaram-se as evidências que indicavam o hábito de Ae. scapularis de exercer a sua hematofagia em fontes alimentares existentes no ambiente alterado pela ação humana.

2 - Em apreciável proporção, as fêmeas desse culicideo persistem na utilização de abrigos pós-prandiais situados no meio extradomiciliar. Contudo, parte delas, e em número aparentemente significativo, encontra condições adequadas para abrigo e repouso no ambiente peridomiciliar.

3 - Repetiu-se a observação que mostra preferência alimentar de Ae. scapularis por sangue de mamíferos. Em especial modo, essa predileção dirige-se a hospedeiros de grande porte como bovinos, equiinos e $o$ próprio homem, mesmo em ambiente com grande número de outros mamíferos e animais domésticos disponíveis.

4 - Nos ambientes alterados que sediaram a realização da pesquisa, o Ae. scapularis foi espécie de presença dominante nas coletas efetuadas e com a maioria de reações positivas aos testes para o diagnóstico do sangue ingerido.

5 - Tornou-se evidente que a presença de animais domésticos no peridomicílio exerce atração, não apenas sobre as fêmeas de Ae. scapularis, mas também sobre as de outros culicídeos, principalmente representantes de Culex (Melanoconion). Dentre estes, destacaram-se $C x$, ribeirensis e $C x$. sacchettae. 
6 - O número de espécies de Melanoconion encontradas no meio peridomiciliar foi considerável, em especial modo nas áreas de intensa atividade agropecuária. A explicação poderá ser encontrada, ao menos parcialmente, na atração exercida pelo grande número de animais domésticos encontrado nesse ambiente.

7 - Dentre as espécies do supracitado subgênero destacou-se $C x$. ribeirensis que, nas presentes observações e a exemplo do que se evidenciou nas anteriores, mostrou nitida tendência para freqüentar o ambiente humano.

8 - Ae. scapularis, Cx. ribeirensis e muito provavelmente outros culicídeos do subgênero Melanoconion apresentam valên- cia ecológica suficiente para lhes permitir evolução de hábitos no sentido da domiciliação.

9 - Os resultados recentemente divulgados, de observações levadas a efeito na região norte da Argentina, mostraram que espécies pertencentes àquela categoria subgenérica, como $C x$. delpontei, desempenham possível papel na veiculação enzoó. tica de alguns arbovírus, entre os quais o da encefalite eqüina venezuelana. A presença desse culicídeo na região estudada, foi registrada nesta e em investigações anteriores. Isso torna oportuna a realização de pesquisas que visem melhor conhecimento dos hábitos desse e de outros representantes do mesmo subgênero. FORATTINI, O.P. et al. [Feeding preferences and domiciliation of Culicidae mosquitoes in the Ribeira
Valley, S. Paulo State, Brazil, with particular reference to Aedes scapularis and Culex (Melanoco-
nion)]. Rev. Saúde públ., S. Paulo, 23:9-19, 1989.

\begin{abstract}
New results on blood-meal identification and the environmental distribution of mosquitoes collected in four different Ribeira Valley (S. Paulo State, Brazil) environments, during the period February to November 1986, are presented. Sources of 651 blood-meals were identified. The preference of Ae. scapularis for large mammals, chiefly cattle, horse and even man, was confirmed. Data suggests that a similar behavioral pattern is presented by $C x$. ribeirensis. Both mosquitoes seem to be strongly attracted by peridomiciliar blood sources represented by domestic animals sheltered in that environment. Nevertheless, the female of Ae. scapularis females may use the extradomiciliary environment, represented by residual forests, as resting place in the same way as it uses the peridomiciliary one. The data confirm the clear tendency of both Ae. scapularis and Cx. ribeirensis to adapt to the man-made environment and thus the evolution towards domiciliation in the behavior of these mosquitoes. Other culicids, such as $A n$. bellator, $A n$. cruzii and Cq. chrysonotum, were collected practically only by the use of human bait. For $A n$. cruzii the number of females collected by this method represented $31.6 \%$ of the female total collected during this project. Some data about other species are given, and the need for further researches about Culex (Melanoconion) species is emphasized.
\end{abstract}

KEYWORDS: Mosquitoes. Food preferences. Aedes scapularis. Culex. Anthropophily. Domiciliation. Insect vectors.

\title{
REFERENCIAS BIBLIOGRÁFICAS
}

1. DEGALLIER, N.; PAJOT, F.-X.; KRAMER, R.; CLAUSTRE, J.; BELLONY, S.; LE PONT, F. Rythmes d'activité des Culicidés de la Guyane française (Diptera, Culicidae). Cah. O.R.S.T.O.M. Ent. méd. parasitol., 16:73-84, 1978.

2. FORATTINI, O.P. \& GOMES, A. de C. Biting activity patterns of Culex (Melanoconion) ribeirensis in Southern Brazil. J. Amer. Mosq. Control Ass., 4:175-8, 1988.

3. FORATTINI, O.P.; GOMES, A. de C.; NATAL, D.; SANTOS, J.L.F. Observaçठ̌es sobre atividade de mosquitos Culicidae em matas primitivas da planicie e perfís epidemiológicos de vários ambientes no Vale do Ribeira, São Paulo, Brasil. Rev. Saúde públ., S. Paulo, 20:178-203, 1986.

4. FORATTINI, O.P.; GOMES, A. de C.; NATAL, D.; KAKITANI, I.; MARUCCI, D. Preferências alimentares de mosquitos Culicidae no Vale do Ribeira,
São Paulo, Brasil. Rev. Saúde públ., S. Paulo, 21:171-87, 1987.

5. FORATTINI, O.P.; GOMES, A. de C.; NATAL, D.; KAKITANI, I.; MARUCCI, D. Frequêencia domiciliar e endofilia de mosquitos Culicidae no Vale do Ribeira, São Paulo, Brasil. Rev. Saúde públ., S. Paulo, 21:188-92, 1987.

6. GUIMARÄES, A.E.; ARLE, M.; MACHADO, R.N.M. Mosquitos no Parque Nacional da Serra dos Orgãos, Estado do Rio de Janeiro, Brasil. IV - Preferência alimentar. Mem. Inst. Oswaldo Cruz, Rio de Janeiro, 82:277-85, 1987.

7. MITCHELL, C.J.; DARSIE Jr., R.F.; MONATH, T.P.; SABATTINI, M.S.; DAFFNER, J. The use of an animal-baited net trap for collecting mosquitoes during western equine encephalitis investigations in Argentina. J. Amer. Mosq. Control Ass., 1:43-7, 1985. 
8. MITCHELL, C.J.; MONATH, M.S.; SABATTINI, M.S.; CROPP, C.B.; DAFFNER, J.F.; CALISHER, C.H.; JAKOB, W.L.; CHRISTENSEN, H.A. Arbovirus investigations in Argentina. II : Arthropod collections and virus isolations from mos. quitoes, 1977-1980. Amer. J. trop. Med. Hyg., 34:945-55, 1985.

9. MITCHELL, C.J.; MONATH, T.P.; SABATTINI, M.S.; CHRISTENSEN, H.A.; DARSIE Jr., R.F.; JAKOB, W.L.; DAFFNER, J.F. Host-feeding patterns of Argentine mosquitoes (Diptera: Culicidae) collected during and after an epizootic of western equine encephalitis. J. med. Ent., 24:260-7, 1987.

10. OLIVEIRA, R.L. de Alguns aspectos da ecologia dos mosquitos (Diptera: Culicidae) em uma área da planície (Granjas Calábria), em Jacarepaguá, Rio de Janeiro. I - Frequência comparativa das espécies em diferentes ambientes e métodos de coleta. Mem. Inst. Oswaldo Cruz, Rio de Janeiro, 79:479-90, 1984.
11. OLIVEIRA, R.L. de \& HEYDEN, R. Alguns aspectos da ecologia dos mosquitos (Diptera: Culicidae) de uma área de planície (Granjas Calábria), em Jacarepaguá, Rio de Janeiro. IV - Preferências alimentares quanto ao hospedeiro e frequência domiciliar. Mem. Inst. Oswaldo Cruz, Rio de Janeiro, 81:15-27, 1986.

12. ROBERTS, D.R.; PEYTON, E.L.; PINHEIRO, F.B.; BALDERRAMA, F.; VARGAS, R. Asociación de vectores de arbovirus con galerias arboreas $y$ el medio doméstico en el sureste de Bolivia. Bol. Ofic. sanit. panamer., 98:417-30, 1985.

13. TEMPELIS, C.H. \& LOFY, M.F. A modified precipitin method for identification of mosquito blood-meals. Amer. J. trop. Med. Hyg., 12:825-31, 1963.

Recebido para publicaça em 18/10/1988. Aprovado para publicaçdo em 22/12/1988. 